TRANSACTIONS OF THE

AMERICAN MATHEMATICAL SOCIETY

Volume 359, Number 7, July 2007, Pages 2993-3004

S 0002-9947(07)03976-1

Article electronically published on February 8, 2007

\title{
DELAUNAY CONFIGURATIONS AND MULTIVARIATE SPLINES: A GENERALIZATION OF A RESULT OF B. N. DELAUNAY
}

\author{
MARIAN NEAMTU
}

\begin{abstract}
In the 1920s, B. N. Delaunay proved that the dual graph of the Voronoi diagram of a discrete set of points in a Euclidean space gives rise to a collection of simplices, whose circumspheres contain no points from this set in their interior. Such Delaunay simplices tessellate the convex hull of these points. An equivalent formulation of this property is that the characteristic functions of the Delaunay simplices form a partition of unity. In the paper this result is generalized to the so-called Delaunay configurations. These are defined by considering all simplices for which the interiors of their circumspheres contain a fixed number of points from the given set, in contrast to the Delaunay simplices, whose circumspheres are empty. It is proved that every family of Delaunay configurations generates a partition of unity, formed by the so-called simplex splines. These are compactly supported piecewise polynomial functions which are multivariate analogs of the well-known univariate B-splines. It is also shown that the linear span of the simplex splines contains all algebraic polynomials of degree not exceeding the degree of the splines.
\end{abstract}

\section{INTRODUCTION}

Let $K$ be a discrete set of points in the $s$-dimensional Euclidean space $\mathbb{R}^{s}, s \geq 1$. In the 1920s, B. N. Delaunay proved that the dual graph of the Voronoi diagram of $K$, now called the Delaunay triangulation of $K$, can be obtained by considering special subsets of $K$ of size $s+1$. The convex hulls of such subsets, called Delaunay simplices, have the characteristic property that their circumspheres contain no points from $K$ in their interior. Moreover, the Delaunay simplices are known to tessellate the convex hull $[K]$ of $K[6$. Voronoi diagrams and Delaunay triangulations are fundamental concepts in computational geometry and have many applications. We refer the reader to [11, 12 for more information and references on this subject.

To explain the main result of this paper, we will give an alternative interpretation of the fact that Delaunay simplices triangulate the convex hull $[K]$. To avoid technical complications in our presentation, we first make some assumptions about the set $K$. Throughout the paper, $K$ will be an infinite set of points such that $[K]=\mathbb{R}^{s}$. Moreover, $K$ will be assumed to be locally finite, i.e., such that $K \cap \Omega$ is finite for every compact $\Omega \subset \mathbb{R}^{s}$. Lastly, the points in $K$ will be assumed to be

Received by the editors February 12, 2004 and, in revised form, February 4, 2005.

2000 Mathematics Subject Classification. Primary 41A15, 41A63; Secondary 05B45, 52C22, 65D17, 65D18.

Key words and phrases. Delaunay configuration, Delaunay triangulation, higher-order Voronoi diagram, multivariate spline, polynomial reproduction, simplex spline.

This work was supported by the NSF under grant CCF-0204174.

(C)2007 American Mathematical Society 2993

Reverts to public domain 28 years from publication 
in generic position in the sense that every subset of size $s+1$ can be circumscribed a unique $s$-dimensional sphere and that no $s+2$ points in $K$ are co-spherical, i.e., lie on a common sphere in $\mathbb{R}^{s}$. The first of these two assumptions is equivalent to the property that all collections of $s+1$ points are affinely independent i.e., do not lie in a common hyperplane. In the bivariate case, the above conditions mean that no three points in $K$ should be collinear and no four points co-circular.

Let $\Delta$ be the Delaunay triangulation of $K$. We will view $\Delta$ as a collection of subsets $X$ of $K$ of size $s+1$, whose circumspheres have no points from $K$ in their interior. Note that the assumption that the points in $K$ are in generic position guarantees that $\Delta$ is well defined. The above-mentioned fact that Delaunay simplices triangulate $[K]=\mathbb{R}^{s}$ can be restated by saying that the characteristic functions of these simplices form a partition of unity. That is,

$$
\sum_{X \in \Delta} \chi_{[X]}(x)=1, \quad \text { a.e. } x \in \mathbb{R}^{s}
$$

where $\chi_{A}$ denotes the characteristic function of a set $A \subset \mathbb{R}^{s}$.

In this paper, we generalize this result by considering a fixed number of points from $K$ to be inside the circumspheres of $s+1$ points, as opposed to no points in the case of Delaunay simplices. We say that a closed ball $\Omega$ in $\mathbb{R}^{s}$ is circumscribed to a set $A$ if $A \subset \partial \Omega$. As usual, int $\Omega$ and $\partial \Omega$ will denote the interior and the boundary of $\Omega$, respectively. If $A$ is a finite set, we denote its size by $\# A$.

Definition 1.1. Let $k \in \mathbb{Z}_{+}^{0}$. The ordered pair $X=\left(X_{B}, X_{I}\right)$ such that $X_{B}, X_{I} \subset$ $K, \# X_{B}=s+1, \# X_{I}=k$, and such that the closed ball $\Omega \subset \mathbb{R}^{s}$ circumscribed to $X_{B}$ contains $X_{I}$ in its interior, and no other points from $K$, i.e., such that $X_{B}=\partial \Omega \cap K$ and $X_{I}=\operatorname{int} \Omega \cap K$, is called a Delaunay configuration of degree $k$. For $k=0$, the set $X_{I}$ is understood to be empty. The family of all Delaunay configurations of degree $k$ associated with the set $K$ will be denoted by $\Delta_{k}$.

The subscript " $B$ " in the definition stands for "boundary", since the points $X_{B}$ lie on the boundary of $\Omega$, whereas the subscript " $I$ " signifies that $X_{I}$ are "interior" points. In the theorem below, a partition of unity will be obtained using piecewise polynomial functions of arbitrary degree $n$. In particular, the role of $\Delta$ will be played by the family of Delaunay configurations $\Delta_{n}$, and the characteristic functions in (1.1) will be replaced with the so-called normalized simplex splines of degree $n$, denoted by $N(\cdot \mid X)$, whose precise definition will be given in Section 3 . These are compactly supported piecewise polynomials, defined by a collection $X$ of $n+s+1$ points in $\mathbb{R}^{s}$, called knots. Such functions are generally smooth, as opposed to the characteristic functions, which are only piecewise constant and are therefore discontinuous along the boundaries of the Delaunay simplices. In fact, if the knots are such that all subsets of $X$ of size $s+1$ are affinely independent, which is our case, the simplex splines $N(\cdot \mid X)$ are optimally smooth, or $N(\cdot \mid X) \in C^{n-1}\left(\mathbb{R}^{s}\right)$.

Theorem 1.2. Let $\Delta_{n}, n \in \mathbb{N}$, be the family of Delaunay configurations of degree $n$ of $K$. Then

$$
\sum_{X \in \Delta_{n}} N(x \mid X)=1, \quad x \in \mathbb{R}^{s}
$$

Note that this is indeed a generalization of (1.1) since $\Delta_{0}=\Delta$ and also since for $n=0$, the functions $N(\cdot \mid X)$ are just the characteristic functions of $[X]$. To prove the theorem, in the next section we will first be concerned with some basic combinatorial 


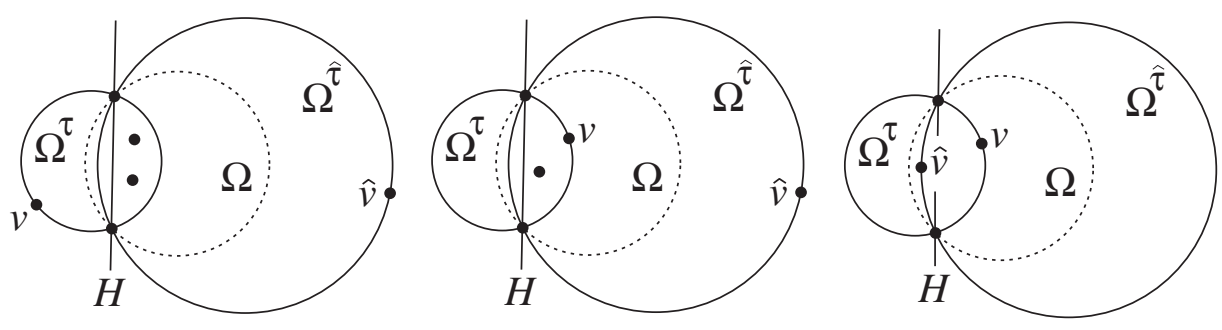

Figure 1. (a) Non-essential, (b) essential, and (c) phantom faces of degree two

properties of Delaunay configurations and, in Section 3, we will review some needed facts about simplex splines. Theorem 1.2 will be a special case of a more general result that the linear span of the simplex splines $N(\cdot \mid X), X \in \Delta_{n}$, contains all polynomials of degree at most $n$. The remainder of the paper is devoted to the proof of this more general result and its discussion.

\section{Delaunay configurations}

In this section we establish a useful structural property of Delaunay configurations, which is a consequence of the following elementary observation. For a set $A$ and elements $u \in A, v \notin A$, we employ the abbreviated notation ${ }_{u} A:=A \backslash\{u\}$, ${ }^{v} A:=A \cup\{v\},{ }_{u}^{v} A:=(A \backslash\{u\}) \cup\{v\}$, and aff $A$ denotes the affine hull of $A$.

Proposition 2.1. Let $k \in \mathbb{Z}_{+}^{0}$ and $F_{B}, F_{I} \subset K, \# F_{B}=s, \# F_{I}=k$, be such that $F_{I}=\operatorname{int} \Omega \cap K$, for some closed ball $\Omega$ circumscribed to $F_{B}$. Then there exist precisely two distinct points $v, \hat{v} \in K \backslash F_{B}$ such that the closed balls circumscribed to ${ }^{v} F_{B}$ and ${ }^{v} F_{B}$ contain the points ${ }^{v} F_{B} \cup F_{I}$ and ${ }^{v} F_{B} \cup F_{I}$, respectively, and no other points from $K$. Moreover, there are only three mutually exclusive possibilities:

(a) $\#\left(\{v, \hat{v}\} \cap F_{I}\right)=0$ and $v, \hat{v}$ are separated by the hyperplane aff $F_{B}$;

(b) \# $\left(\{v, \hat{v}\} \cap F_{I}\right)=1$ and $v, \hat{v}$ are on the same side of aff $F_{B}$;

(c) $\#\left(\{v, \hat{v}\} \cap F_{I}\right)=2$ and $v, \hat{v}$ are separated by aff $F_{B}$.

Figure 1 shows the three possibilities (a)-(c) in two dimensions. The notation in this figure is defined in the proof of the proposition, and the terminology used in the caption will be explained in Definition 2.2 below. The dots represent the points in $K$, where it is understood that the remaining points of $K$ are outside the union of the depicted disks. We remark that for $k=0$, only the case (a) can occur, whereas for $k=1$, only (a) and (b) are possible.

Proof. Let $H:=\operatorname{aff} F_{B}$. By our assumption that all collections of $s+1$ points in $K$ are affinely independent, so is every subset of $s$ points, hence $H$ is a hyperplane in $\mathbb{R}^{s}$. Let $H^{+}$and $H^{-}$be the open halfspaces determined by $H$. Consider the set of all closed balls circumscribed to $F_{B}$. It is easy to see that this set is a continuous one-parameter family. We denote this family by $\left\{\Omega^{t}\right\}$ and coordinatize it by the straight line traced out by the centers of these balls, parametrized by a parameter $t \in \mathbb{R}$. Note that this line is perpendicular to $H$ and passes through the circumcenter of the points $F_{B}$ in $H$. We let the circumcenter be the origin of the coordinate system along this line and choose the orientation of this line such that $H^{+}$corresponds to positive values of $t$, in the sense that the center of $\Omega^{t}, t>0$, 
is in $H^{+}$. Also, let $T:=\left\{t \in \mathbb{R} \mid F_{I} \subset \Omega^{t}, K \cap\right.$ int $\left.\Omega^{t} \subset F_{I}\right\}$. Since $H^{+} \subset \bigcup_{t>0} \Omega^{t}$ and $H^{-} \subset \bigcup_{t<0} \Omega^{t}$, it follows from the assumption $[K]=\mathbb{R}^{s}$ that $T$ is bounded. Moreover, it can be shown that $T$ is a closed interval, which we denote by $[\tau, \hat{\tau}]$. We will dispense with the proof of this simple observation. The fact that $\tau<\hat{\tau}$ follows from $\Omega \in\left\{\Omega^{t}\right\}_{t \in \operatorname{int} T}$. Lastly, it is not difficult to show that $\Omega^{t} \backslash H \subset \operatorname{int} \Omega^{\tau} \cup \operatorname{int} \Omega^{\hat{\tau}}$ and $\Omega^{\tau} \cap \Omega^{\hat{\tau}} \subset$ int $\Omega^{t} \cup H$, whenever $t \in(\tau, \hat{\tau})$.

Proving the first part of the proposition is equivalent to showing that there are precisely two points $v \in K \backslash F_{B}$ such that $v \in \partial \Omega^{t}$, for some $t \in[\tau, \hat{\tau}]$. Clearly, $v \notin$ $H$, for then the points in ${ }^{v} F_{B}$ would be affinely dependent. The above observations now imply that we cannot have $t \in(\tau, \hat{\tau})$, for then $v \in \Omega^{t} \backslash H \subset \operatorname{int} \Omega^{\tau} \cup \operatorname{int} \Omega^{\hat{\tau}}$ and hence $v \in F_{I} \subset \Omega^{\tau} \cap \Omega^{\hat{\tau}} \subset$ int $\Omega^{t} \cup H$, which would contradict the fact that $v \in \partial \Omega^{t}$. Thus, $t$ can only be one of the endpoints of the interval $[\tau, \hat{\tau}]$. Therefore, let $t=\tau$ and let $v \in \partial \Omega^{\tau} \cap K \backslash F_{B}$. Such a point exists, for otherwise $K \cap \partial \Omega^{\tau}=F_{B}$ and hence $F_{I} \subset \operatorname{int} \Omega^{\tau}$. Consequently, $\tau \in \operatorname{int} T$, which is a contradiction. The uniqueness of $v$ follows from the fact that no $s+2$ points from $K$ are co-spherical. By a similar argument, there exists a unique point $\hat{v} \in \partial \Omega^{\hat{\tau}} \cap K \backslash F_{B}$.

To prove the remaining part of the proposition, suppose that $v$ and $\hat{v}$ are not separated by $H$ and assume without loss of generality that $v, \hat{v} \in H^{-}$. It is not difficult to show that $\Omega^{\hat{\tau}} \cap H^{-} \subset \operatorname{int} \Omega^{\tau}$, which implies $\hat{v} \in \Omega^{\hat{\tau}} \cap H^{-} \subset \operatorname{int} \Omega^{\tau}$, i.e., $\hat{v} \in F_{I}$. Moreover, this also means $v \notin F_{I}$, since otherwise $v \in F_{I} \subset \Omega^{\hat{\gamma}} \cap H^{-} \subset$ int $\Omega^{\tau}$, which would contradict the fact that $v \in \partial \Omega^{\tau}$. This shows that if $v$ and $\hat{v}$ are not separated, then only case (b) can occur. Conversely, we show that if precisely one of the points $v, \hat{v}$ is in $F_{I}$, then both $v$ and $\hat{v}$ must be on the same side of $H$. To see this, assume without loss of generality that $v \in H^{-}$and $\hat{v} \in H^{+}$, and first consider the case where $v \in F_{I}$ and $\hat{v} \notin F_{I}$. Then $v \in F_{I} \subset \Omega^{\hat{\gamma}}$ and therefore $v \in \Omega^{\hat{\tau}} \cap H^{-} \subset \operatorname{int} \Omega^{\tau}$, which contradicts the fact that $v \in \partial \Omega^{\tau}$. In a similar way one can arrive at a contradiction in the case where $v \notin F_{I}$ and $\hat{v} \in F_{I}$. Thus, we can conclude that $v$ and $\hat{v}$ must be on the same side of $H$, which finishes the proof.

The above proposition suggests the following definition.

Definition 2.2. Let $k \in \mathbb{Z}_{+}^{0}, F=\left(F_{B}, F_{I}\right)$ be an ordered pair of subsets of $K$ satisfying the assumptions of Proposition 2.1, and let $v, \hat{v}$ be the associated points whose existence is guaranteed by this proposition. We say that $F$ is a non-essential face of degree $k$, if $v, \hat{v}$ are not contained in $F_{I}$, and an essential face of degree $k$, if precisely one of the points $v, \hat{v}$ belongs to $F_{I}$. Otherwise, if $v, \hat{v} \in F_{I}, F$ is called a phantom face of degree $k$. The set of all essential faces of degree $k$ will be denoted by $\mathcal{F}_{k}^{e}$. If $X=\left(X_{B}, X_{I}\right) \in \Delta_{k}$ and $F=\left(F_{B}, F_{I}\right)=\left({ }_{v} X_{B}, X_{I}\right)$, for some $v \in X_{B}$, we say that $F$ is a face of $X$.

A few remarks on this definition are in order. The definition says that the pair $F$ is a non-essential, essential, and phantom face if properties (a), (b), and (c) of Proposition 2.1 are satisfied, respectively. Also, essential and non-essential faces can be interpreted as faces of Delaunay configurations. In particular, since for such faces at least one of the points $v, \hat{v}$, say $v$, does not belong to $F_{I}$, it follows that $X:=\left({ }^{v} F_{B}, F_{I}\right) \in \Delta_{k}$, i.e., $F$ is a face of $X$. The difference between essential and non-essential faces is that for non-essential faces $F$, the configuration $\hat{X}:=\left({ }^{\hat{v}} F_{B}, F_{I}\right)$ is also of degree $k$, while $\hat{X}$ is of degree $k-1$ if $F$ is essential. For phantom faces of degree $k$, both $X$ and $\hat{X}$ are of degree $k-1$. This means that a face of a Delaunay 
configuration of degree $k$ can only be either essential or non-essential, i.e., it cannot be a phantom face, hence the denomination "phantom" since it is not a face of an actual configuration. We can conclude that each essential face corresponds to a pair of Delaunay configurations of different degrees and that non-essential and phantom faces are associated with pairs of configurations of the same degree.

\section{Simplex splines}

Having defined a generalization of a Delaunay triangulation, we also need an analog of the characteristic function of a simplex. For our purpose, such suitable analogs are the so-called simplex splines. These are multivariate generalizations of the well-known univariate B-splines of Schoenberg [3]. They were defined by de Boor in 1976 [2], who was inspired by a suggestion of Schoenberg; see 9]. The origins of simplex splines can also be traced back to statisticians [4].

We briefly recall some basic facts about simplex splines. For a more detailed explanation, see [9] and the references therein. Let $X=\left\{x^{i}\right\}_{i=0}^{n+s}$ be a set of $n+s+1$ points in $\mathbb{R}^{s}$, called knots in spline theory. The simplex spline with knots $X$ can be defined as a distribution, denoted by $M(\cdot \mid X)$, satisfying the identity

$$
\int_{\mathbb{R}^{s}} M(x \mid X) f(x) d x=n ! s ! \int_{S^{n+s}} f\left(\sum_{i=0}^{n+s} t_{i} x^{i}\right) d t_{1} \cdots d t_{n+s},
$$

for all $f \in L_{1}\left(\mathbb{R}^{s}\right)$, where

$$
S^{n+s}:=\left\{\left(t_{0}, \ldots, t_{n+s}\right) \mid \sum_{i=0}^{n+s} t_{i}=1, t_{0} \geq 0, \ldots, t_{n+s} \geq 0\right\}
$$

denotes the standard simplex in $\mathbb{R}^{n+s}$. If the knots $X$ do not all lie in a single hyperplane, the simplex spline is known to be a non-negative piecewise polynomial function of degree $n$, supported on the convex hull $[X]$ of $X$. For $s=1, M$ is the familiar univariate B-spline [15]. If the knots are in general position, in the sense that any subset of $s+1$ knots from $X$ are affinely independent, $M$ is $n-1$ times continuously differentiable, i.e., it is of optimal smoothness. Moreover, for $n=0$, or $\# X=s+1, M$ is just a renormalized characteristic function of the simplex $[X]$. Specifically,

$$
M(x \mid X)=\frac{\chi_{[X]}(x)}{\operatorname{vol}_{s}[X]}= \begin{cases}1 / \operatorname{vol}_{s}[X], & x \in[X], \\ 0, & \text { otherwise, }\end{cases}
$$

where $\operatorname{vol}_{s} A$ denotes the $s$-dimensional volume of a set $A$.

The numerical evaluation of a simplex spline can be facilitated by a recurrence relation, first established by Micchelli in 1979 [8. The recurrence allows one to compute a simplex spline as a linear combination of lower-degree simplex splines. Here, we only describe a special case of this recursion. To this end, for a non-degenerate simplex in $\mathbb{R}^{s}$ with vertices $V$, we denote by $d(V)$ its oriented $s$-dimensional volume. In particular, if the elements of $V$ are $v^{0}, \ldots, v^{s}$, we can write

$$
d(V):=\frac{1}{s !} \operatorname{det}\left(\begin{array}{ccc}
v_{1}^{0} & \cdots & v_{1}^{s} \\
\vdots & & \vdots \\
v_{s}^{0} & \cdots & v_{s}^{s} \\
1 & \cdots & 1
\end{array}\right)
$$


where $v_{j}^{i}$ denotes the $j$ th coordinate of vertex $v^{i}$. The mentioned special case of Micchelli's recursion now reads as follows.

Proposition 3.1. Let $n \in \mathbb{N}$ and $V \subset X$ be such that $\# V=s+1$ and $d(V) \neq 0$. Then

$$
M(x \mid X)=\sum_{v \in V} \frac{d\left({ }_{v}^{x} V\right)}{d(V)} M\left(\left.x\right|_{v} X\right), \quad \text { a.e. } x \in \mathbb{R}^{s},
$$

where ${ }_{v}^{x} V$ denotes the (ordered) set obtained from $V$ by replacing its element $v$ with the point $x$.

The reason why we wrote that the above identity holds for almost every $x$, instead of every $x$, has to do with the definition (3.1) of lowest-degree (piecewise constant) simplex splines on the boundary of their support. More precisely, if $x$ is strictly inside or outside of every $s$-simplex whose vertices are elements of $X$, then identity (3.2) holds. Otherwise, the point $x$ lies on a lower-dimensional face of a simplex formed by vertices of $X$. As a result, equality in (3.2) may not hold unless the characteristic function of that simplex is defined appropriately on its boundary, as is done, for example, in [16. However, we will refrain from delving into this point, since the precise way that this can be done is of little consequence for us in this paper.

We also remark in passing that the coefficients $d\left({ }_{v}^{x} V\right) / d(V)$ in the recursion are just the barycentric coordinates of $x$ with respect to the knots $V$. The notation ${ }_{v}^{x} V$ suggests that at times it will be important to view a given symbol as a set and other times as an ordered collection. For example, if $X=\left(X_{B}, X_{I}\right)$ is a Delaunay configuration, it is essential to view $X$ as an ordered pair, whereas in the context of the simplex spline $M(\cdot \mid X), X$ can be viewed simply as the set of knots $X_{B} \cup X_{I}$. On the other hand, as we shall see, ordering of the knots $X$ will become important when we consider a specific normalization of $M(\cdot \mid X)$. Since it would be very burdensome to keep separate symbols for ordered and unordered sets, our notation will not distinguish between these two types. However, it will be clear from the context whether the ordering is relevant or not.

\section{Reproduction of POLYNomials}

As follows from their definition and from the above discussion, Delaunay configurations of degree $n$ can be viewed as collections of point-sets of size $n+s+1$. At the same time, an $s$-variate simplex spline with $n+s+1$ knots is also of (polynomial) degree $n$. This suggests that we consider the linear space $\mathcal{S}_{n}$ spanned by the simplex splines whose knot-sets are Delaunay configurations, that is,

$$
\mathcal{S}_{n}:=\operatorname{span}\left\{M(\cdot \mid X) \mid X \in \Delta_{n}\right\},
$$

where, as before, $M(\cdot \mid X)$ is understood as a shorthand notation for $M\left(\cdot \mid X_{B} \cup X_{I}\right)$. We will show in this section that such a space has the interesting property that it contains all polynomials of degree $n$, which is the highest possible degree attainable by splines of degree $n$. The proof of this result will be constructive. We will express any given polynomial explicitly as a linear combination of appropriately normalized simplex splines. Theorem 1.2 will then be a direct consequence of this more general result. 
Let $X=\left(X_{B} X_{I}\right) \in \Delta_{n}$. We will assume throughout that the sets $X_{B}$ are always ordered in such a way that $d\left(X_{B}\right)>0$. We now define a normalized simplex spline of degree $n$ as

$$
N(\cdot \mid X):=d\left(X_{B}\right) M(\cdot \mid X) .
$$

Thus, the above ordering convention guarantees that the normalized simplex splines are nonnegative functions. Also note that for $n=0$, the normalized simplex spline is just the characteristic function of the simplex $\left[X_{B}\right]$, i.e., $N(\cdot \mid X)=\chi_{\left[X_{B}\right]}(\cdot)$, since clearly $d\left(X_{B}\right)=\operatorname{vol}_{s}\left[X_{B}\right]$.

To formulate the main result, we recall the notion of a polar form of an algebraic polynomial. Polar forms, while well known in an algebraic context for quite some time, have been introduced into the spline theory only relatively recently by de Casteljau and independently by Ramshaw (see [13, for a comprehensive introduction). Given an $s$-variate polynomial $p$ of total degree at most $n$, i.e., $p \in \Pi_{n}$, the polar form $P$ of $p$ is defined as the unique function of $n$ vector variables $x^{1}, \ldots, x^{n} \in \mathbb{R}^{s}$, that is symmetric, multi-affine (i.e., affine in each of the $n$ variables), and such that for every $x \in \mathbb{R}^{s}$ the value $p(x)$ is equal to $P$ on the diagonal $x^{1}=\ldots=x^{n}=x$, or $P\left(x^{1}, \ldots, x^{n}\right)=p(x)$. If $n=0$, then $p$ is a constant and $P$ is defined to be this same constant function.

We point out that the above-mentioned multi-affine property can be expressed explicitly as

$$
P\left(\ldots, x^{i}, \ldots\right)=\sum_{v \in V} \frac{d\left(\begin{array}{c}
x^{i} \\
v
\end{array}\right)}{d(V)} P(\ldots, v, \ldots),
$$

which must hold whenever $i \in\{1, \ldots, n\}$ is a fixed index and $V$ is any set of $s+1$ affinely independent points in $\mathbb{R}^{s}$. The dots in the above identity mean that the corresponding vector variables $x^{j}, j \neq i$, are held fixed on both sides of the equation. Thus, identity (4.1) shows that $P$ is affine in the $i$ th variable, since the fractions on the right-hand side are just the barycentric coordinates of $x^{i}$ with respect to the points in $V$.

Theorem 4.1. Let $p \in \Pi_{n}, n \in \mathbb{N}$, and let $P$ be the polar form of $p$. Then

$$
p(x)=\sum_{X \in \Delta_{n}} P\left(X_{I}\right) N(x \mid X), \quad x \in \mathbb{R}^{s} .
$$

Setting $p \equiv 1$ or, equivalently, $P \equiv 1$, we see that Theorem 1.2 follows immediately as a special case of Theorem 4.1 Note that using recurrence (3.2), the proof of this theorem would be straightforward if all "faces" of the normalized simplex splines $N$, i.e., all simplex splines whose knot-sets are faces of $X$, corresponded to lower-degree configurations in $\Delta_{n-1}$ and, at the same time, if all simplex splines associated with $\Delta_{n-1}$ corresponded to faces of configurations of degree $n$. This is precisely the property that can be used to prove the univariate analog of (4.2) and can be attributed to the fact that in one spatial dimension all faces are essential. However, for $s \geq 2$, the simplex splines no longer have this property. Namely, as follows from Section 2, non-essential faces do not give rise to lower-degree configurations and lower-degree configurations associated with phantom faces do not beget higher-degree configurations. Nonetheless, these cases can be handled well since, as we have seen, non-essential and phantom faces are associated with configurations that come in pairs. Owing to this fact, the cumulative effect of these pairs 
in representing polynomials will be null. This will allow us to employ an inductive argument in the proof.

Proof. We divide the proof into several parts. First we prove that for every $k \in \mathbb{N}$, the following identity holds:

$$
\sum_{X \in \Delta_{k}} Q\left(X_{I}\right) N(x \mid X)=\sum_{X^{\prime} \in \Delta_{k-1}} Q\left(X_{I}^{\prime}, x\right) N\left(x \mid X^{\prime}\right), \quad \text { a.e. } x \in \mathbb{R}^{s},
$$

where $Q$ is the polar form of an arbitrary polynomial $q \in \Pi_{k}$. The proof will then be completed by induction. For the remainder of the proof, unless said otherwise, all equalities will be understood to hold for a.e. $x$.

(1) Using recurrence (3.2), we obtain

$$
\begin{aligned}
\sum_{X \in \Delta_{k}} Q\left(X_{I}\right) N(x \mid X) & =\sum_{X \in \Delta_{k}} Q\left(X_{I}\right) d\left(X_{B}\right) \sum_{v \in X_{B}} \frac{d\left(\begin{array}{l}
x \\
v
\end{array} X_{B}\right)}{d\left(X_{B}\right)} M\left(\left.x\right|_{v} X\right) \\
& =\sum_{X \in \Delta_{k}} Q\left(X_{I}\right) \sum_{v \in X_{B}} d\left({ }_{v}^{x} X_{B}\right) M\left(\left.x\right|_{v} X\right) .
\end{aligned}
$$

Thus, the linear combination on the left-hand side of (4.4) is written as a combination of lower-degree simplex splines of the form $M(\cdot \mid F)$, where $F={ }_{v} X:=$ $\left({ }_{v} X_{B}, X_{I}\right)$ are the faces of configurations $X \in \Delta_{k}$. Proposition 2.1 asserts that if a face of degree $k$ of the form $F={ }_{v} X$ is non-essential, then there exists exactly one other Delaunay configuration $\hat{X} \in \Delta_{k}$ and a $\hat{v} \in \hat{X}_{B}$ on the opposite side of the hyperplane aff $F_{B}=\operatorname{aff}_{v} X_{B}=\operatorname{aff}_{\hat{v}} \hat{X}_{B}$ as $v$, such that $F$ is also a face of $\hat{X}$, or $F=\hat{v} \hat{X}$. Thus, the contribution of $X$ and $\hat{X}$ to (4.4) is

$$
Q\left(X_{I}\right) d\left({ }_{v}^{x} X_{B}\right) M(x \mid F)+Q\left(\hat{X}_{I}\right) d\left({ }_{\hat{v}}^{x} \hat{X}_{B}\right) M(x \mid F),
$$

which is zero since $X_{I}=\hat{X}_{I}$ and $d\left({ }_{v}^{x} X_{B}\right)=-d\left({ }_{\hat{v}}^{x} \hat{X}_{B}\right)$, a consequence of $v$ and $\hat{v}$ being separated by aff $F_{B}$ and the ordering convention $d\left(X_{B}\right), d\left(\hat{X}_{B}\right)>0$. Thus, only essential faces can contribute non-trivially to (4.4). Moreover, each essential face $F={ }_{v} X$ appears in (4.4) exactly once since by Proposition 2.1 there exists only one point $v$ such that ${ }^{v} F \in \Delta_{k}$. Therefore, denoting this point by $v_{F}:=v$, (4.4) can be rewritten as

$$
\sum_{X \in \Delta_{k}} Q\left(X_{I}\right) N(x \mid X)=\sum_{F \in \mathcal{F}_{k}^{e}} Q\left(F_{I}\right) d\left({ }_{v_{F}}^{x}\left(v_{F} F_{B}\right)\right) M(x \mid F),
$$

where we follow the convention that the orderings of the collections $X_{B}$ and ${ }^{v_{F}} F_{B}$, which are identical as sets, are the same, so that $d\left({ }^{v_{F}} F_{B}\right)>0$.

(2) We now consider the right-hand side of (4.3). Using the multi-affinity (4.1) of the polar form $Q$, we can write

$$
\begin{aligned}
\sum_{X^{\prime} \in \Delta_{k-1}} Q\left(X_{I}^{\prime}, x\right) N\left(x \mid X^{\prime}\right) & =\sum_{X^{\prime} \in \Delta_{k-1}} N\left(x \mid X^{\prime}\right) \sum_{v \in X_{B}^{\prime}} \frac{d\left(\begin{array}{l}
x \\
v
\end{array} X_{B}^{\prime}\right)}{d\left(X_{B}^{\prime}\right)} Q\left(X_{I}^{\prime}, v\right) \\
& =\sum_{X^{\prime} \in \Delta_{k-1}} M\left(x \mid X^{\prime}\right) \sum_{v \in X_{B}^{\prime}} d\left(\begin{array}{l}
x \\
v
\end{array} X_{B}^{\prime}\right) Q\left(X_{I}^{\prime}, v\right) .
\end{aligned}
$$

Let $F^{\prime}={ }_{v} X^{\prime}, v \in X_{B}^{\prime}$, be any face of $X^{\prime}$ and let $F:=\left(F_{B}^{\prime},{ }^{v} F_{I}^{\prime}\right)$. Clearly, $F$ cannot be a non-essential face of degree $k$ since $X^{\prime}=\left({ }^{v} F_{B},{ }_{v} F_{I}\right)$ is contained in $\Delta_{k-1}$, rather than $\Delta_{k}$. Next, suppose that $F$ is a phantom face of degree $k$. By Proposition 2.1, there exists a unique point $\hat{v} \in{ }_{v} F_{I}=F_{I}^{\prime}$ on the opposite side of 
aff $F_{B}=\operatorname{aff} F_{B}^{\prime}$ as $v$, such that $\hat{X}^{\prime}:=\left({ }^{\hat{v}} F_{B}, \hat{v} F_{I}\right) \in \Delta_{k-1}$. Thus, the contribution to (4.6) of the simplex splines with knots $X^{\prime}$ and $\hat{X}^{\prime}$ is

$$
M\left(x \mid X^{\prime}\right) d\left({ }_{v}^{x}\left({ }^{v} F_{B}^{\prime}\right)\right) Q\left(X_{I}^{\prime}, v\right)+M\left(x \mid \hat{X}^{\prime}\right) d\left({ }_{\hat{v}}^{x}\left(\hat{v} F_{B}^{\prime}\right)\right) Q\left(\hat{X}_{I}^{\prime}, \hat{v}\right)=0 .
$$

This is because $X^{\prime}$ and $\hat{X}^{\prime}$ are identical as sets, $d\left({ }_{v}^{x}\left({ }^{v} F_{B}^{\prime}\right)\right)=-d\left({ }_{\hat{v}}^{x}\left(\hat{v} F_{B}^{\prime}\right)\right)$, since $v$ and $\hat{v}$ are separated by aff $F_{B}^{\prime}$, and $Q\left(X_{I}^{\prime}, v\right)=Q\left(\hat{X}_{I}^{\prime}, \hat{v}\right)$, by the fact that $X_{I}^{\prime} \cup\{v\}=$ $\hat{X}_{I}^{\prime} \cup\{\hat{v}\}$ and by the symmetry of $Q$. Note also that above we invoked the convention that ${ }^{v} F_{B}^{\prime}$ and ${ }^{\hat{v}} F_{B}^{\prime}$ are ordered such that $d\left({ }^{v} F_{B}^{\prime}\right), d\left({ }^{\hat{v}} F_{B}^{\prime}\right)>0$.

The above reasoning leads to the conclusion, similar to part (1) of the proof, that only essential faces $F$ of degree $k$ contribute to (4.6). More precisely, if $F$ is an essential face of the form $F=\left({ }_{v} X_{B}^{\prime},{ }^{v} X_{I}^{\prime}\right)$, for some $X^{\prime} \in \Delta_{k-1}$ and some $v \in X_{B}^{\prime}$, then such $X^{\prime}$ and $v$ are determined uniquely by $F$, for otherwise $F$ would be a phantom face. Consequently, denoting by $v_{F}^{\prime}$ the above unique point $v$ determined by $F$, the sum (4.6) can be rewritten as

$$
\sum_{X^{\prime} \in \Delta_{k-1}} Q\left(X_{I}^{\prime}, x\right) N\left(x \mid X^{\prime}\right)=\sum_{F \in \mathcal{F}_{k}^{e}} Q\left(F_{I}\right) d\left(\begin{array}{l}
x \\
v_{F}^{\prime}
\end{array}\left(v_{F}^{\prime} F_{B}\right)\right) M(x \mid F) .
$$

Finally, since $F$ is essential, we know by Proposition 2.1 that there also exists a unique $v_{F} \in K \backslash F$ such that $\left({ }^{v_{F}} F_{B}, F_{I}\right) \in \Delta_{k}$. Moreover, the points $v_{F}^{\prime}$ and $v_{F}$ are on the same side of aff $F_{B}$. Hence, one has $d\left(x_{v_{F}^{\prime}}^{x}\left(v_{F}^{\prime} F_{B}\right)\right)=d\left({ }_{v_{F}}^{x}\left({ }^{v_{F}} F_{B}\right)\right)$, which shows that

$$
\left.\sum_{F \in \mathcal{F}_{k}^{e}} Q\left(F_{I}\right) d\left({ }_{v_{F}}^{x}\left({ }^{v_{F}} F_{B}\right)\right) M(x \mid F)=\sum_{F \in \mathcal{F}_{k}^{e}} Q\left(F_{I}\right) d\left({ }_{v_{F}^{\prime}}^{x}{ }^{v_{F}^{\prime}} F_{B}\right)\right) M(x \mid F) .
$$

This completes the proof of identity (4.3).

(3) We now return to the assertion of the theorem. Let $n \in \mathbb{N}$. We note that for any $1 \leq k \leq n$ and any fixed $x$, the function

$$
Q\left(y^{1}, \ldots, y^{k}\right):=P(y^{1}, \ldots, y^{k}, \underbrace{x, \ldots, x}_{n-k}), \quad y^{1}, \ldots, y^{k} \in \mathbb{R}^{s},
$$

can be viewed as the polar form of the polynomial $q \in \Pi_{k}$, defined as

$$
q(y):=Q(\underbrace{y, \ldots, y}_{k}) .
$$

Thus, employing identity (4.3) repeatedly (with $k=n, \ldots, 1$ ), we obtain

$$
\begin{aligned}
\sum_{X \in \Delta_{n}} P\left(X_{I}\right) N(x \mid X) & =\sum_{X^{\prime} \in \Delta_{n-1}} P\left(X_{I}^{\prime}, x\right) N\left(x \mid X^{\prime}\right) \\
& =\cdots=\sum_{X^{(n)} \in \Delta_{0}} P(\underbrace{x, \ldots, x}_{n}) N\left(x \mid X^{(n)}\right) .
\end{aligned}
$$

By the diagonal property of $P$, identity (1.1), and the fact that $N\left(\cdot \mid X^{(n)}\right)$ is just the characteristic function of the simplex $\left[X^{(n)}\right]$, the above sum equals

$$
p(x) \sum_{X^{(n)} \in \Delta_{0}} \chi_{\left[X^{(n)}\right]}(x)=p(x) .
$$

Thus, we have shown that identity (4.2) holds true for a.e. $x$. However, since for $n \geq 1$ both sides of (4.2) are continuous functions, by the assumption that the 
set $K$ contains points in generic position, it follows that the identity is in fact true for all $x \in \mathbb{R}^{s}$. This finishes the proof.

It will be interesting to discuss the special situation $s=1$. Suppose that $K \subset \mathbb{R}$ is an increasing bi-infinite sequence of distinct values $\left\{x_{i}\right\}_{i \in \mathbb{Z}}$ such that $[K]=\mathbb{R}$. Clearly, in this case Delaunay configurations $X$ of degree $n$ are of the form $X=\left(X_{B}, X_{I}\right):=\left(\left\{x_{i}, x_{i+n+1}\right\},\left\{x_{i+1}, \ldots, x_{i+n}\right\}\right), i \in \mathbb{Z}$, which means that they are associated with collections of $n+2$ consecutive knots from $K$. For such configurations, the normalized simplex splines are easily seen to be

$$
N(\cdot \mid X)=N\left(\cdot \mid x_{i}, \ldots, x_{i+n+1}\right)=\left(x_{i+n+1}-x_{i}\right) M\left(\cdot \mid x_{i}, \ldots, x_{i+n+1}\right) .
$$

This shows that $N$ is just the well-known normalized B-spline of degree $n$ [15]. Hence, our construction reduces to the familiar univariate construction of B-splines. In the univariate setting, formula (4.2) also has a counterpart; see 13. In this case identity (4.2) becomes

$$
p(x)=\sum_{i \in \mathbb{Z}} P\left(x_{i+1}, \ldots, x_{i+n}\right) N\left(x \mid x_{i}, \ldots, x_{i+n+1}\right), \quad x \in \mathbb{R},
$$

where $p$ is a univariate polynomial of degree $n$ and $P$ is its polar form.

\section{Discussion}

Our interest in the subject of this paper was sparked by the intriguing question of finding multivariate generalizations of univariate splines and, in particular, the problem of defining spline spaces using collections of simplex splines. The motivation for the approach presented here came from an idea of Sabin [14, who suggested that the so-called higher-order Voronoi diagrams, introduced in 1976 by Shamos and Hoey [17, might be utilized in defining appropriate knot-sets for simplex splines. While, as it turned out, such Voronoi diagrams do not lead directly to a suitable construction of spline spaces [10, they are closely related to the Delaunay configurations introduced in this paper. Indeed, the results of this paper show that there is an intimate connection between Delaunay configurations and simplex splines. This connection suggests, on the one hand, that simplex splines are natural geometrical objects and, on the other, that Delaunay configurations and related concepts from computational geometry should be given serious consideration by spline theorists.

The notion of a Delaunay configuration as a separate object of study seems to be new, even though it is implicit in papers concerned with the computation of higher-order Voronoi diagrams. In particular, it has been observed (see e.g., 7, 1] and the books [11, 12]) that collections of points whose circumspheres contain a fixed number of points in their interior play an important role in algorithms for generating higher-order Voronoi diagrams. For example, the notion of a Delaunay tree [1] can be viewed as a hierarchy of Delaunay configurations of varying degrees. The paper [1] in fact contains the observation in the first part of Proposition 2.1. concerning the existence of points $v$ and $\hat{v}$.

The construction of the spline space $\mathcal{S}_{n}$, as defined in Section 4 , is by no means the only possibility of defining a space using simplex splines. In fact, several meaningful constructions of such spaces have been proposed in the literature, notably the construction [5]; see also the survey [10]. Unlike in our case, however, these 
alternative constructions generally do not reduce to the classical splines in one dimension. This means, in view of the discussion following the proof of Theorem 4.1, that our construction is strictly different from these earlier constructions.

In this paper we also demonstrated that polynomials of optimal degree can be represented as linear combinations of simplex splines. As is well known in approximation theory, this so-called polynomial reproduction property is the sine qua

non in the consideration of approximation order of spline spaces. Without being able to represent polynomials, the spline space cannot be expected to have optimal approximation properties. We postpone a discussion of this and other related issues, including the connection between higher-order Voronoi diagrams and Delaunay configurations, the efficient computation of Delaunay configurations, and approximation-theoretic questions pertaining to the new spline space, to another occasion.

\section{ACKNOWLEDGMENTS}

I am indebted to Dr. Bernd Mulansky from the Technical University of Clausthal, for his constructive criticism and a number of suggestions that considerably improved the manuscript.

\section{REFERENCES}

1. Boissonnat, J.-D., O. Devillers, and M. Teillaud, A semidynamic construction of higher-order Voronoi diagrams and its randomized analysis, Algorithmica 9 (1993), 329-356. MR1208566 (94k:68182)

2. Boor, C. de, Splines as linear combinations of B-splines. A survey, in Approximation Theory, II, G. G. Lorentz, C. K. Chui, and L. L. Schumaker (eds.), Academic Press, New York, 1976, 1-47. MR0467092 (57:6959)

3. Curry, H. B., and I. J. Schoenberg, On Pólya frequency functions IV: The fundamental spline functions and their limits, J. Analyse Math. 17 (1966), 71-107. MR0218800 (36:1884)

4. Dahmen, W., and C. A. Micchelli, Statistical encounters with B-splines, in Function Estimates (Arcata, Calif., 1985), Contemp. Math., 59, Amer. Math. Soc., Providence, 1986, 17-48. MR 0870446 (88d:65022)

5. Dahmen, W., C. A. Micchelli, and H.-P. Seidel, Blossoming begets B-splines built better by B-patches, Math. Comp. 59 (1992), 97-115. MR1134724 (93b:41014)

6. Delaunay, B., Sur la sphère vide, Bull. Acad. Sci. USSR (VII), Classe Sci. Mat. Nat., 1934, 793-800.

7. Lee, D.-T., On $k$-nearest neighbor Voronoi diagrams in the plane, IEEE Trans. Comp. 31 (1982), 478-487. MR0669964 (83m:68157)

8. Micchelli, C. A., A constructive approach to Kergin interpolation in $\mathbb{R}^{k}$ : multivariate B-splines and Lagrange interpolation, Rocky Mountain J. Math. 10 (1980), 485-497. MR0590212 (84i:41002)

9. Micchelli, C. A., Mathematical Aspects of Geometric Modeling, CBMS-NSF Regional Conference Series in Applied Mathematics, 65, SIAM, Philadelphia, 1995. MR1308048 (95i:65036)

10. Neamtu, M., What is the natural generalization of univariate splines to higher dimensions?, in Mathematical Methods for Curves and Surfaces: Oslo 2000, T. Lyche and L. L. Schumaker (eds.), Vanderbilt University Press, Nashville, TN, 2001, 355-392. MR1858970

11. Okabe, A., B. Boots, and K. Sugihara, Spatial Tessellations: Concepts and Applications of Voronoi Diagrams, 2nd ed., Wiley, Chichester, England, 2000. MR.1770006 (2001c:52013)

12. Preparata, F. P., and M. I. Shamos, Computational Geometry, Springer, 1985. MR0805539 (87d:68102)

13. Ramshaw, L., Blossoms are polar forms, Comput. Aided Geom. Design 6 (1989), 323-358. MR 1030618 (91d:65026)

14. Sabin, M., Open questions in the application of multivariate B-splines, in Mathematical Methods in Computer Aided Geometric Design, T. Lyche and L. L. Schumaker (eds.), Academic Press, New York, 1989, 529-537. MR1022732 (90k:65050) 
15. Schumaker, L. L., Spline Functions: Basic Theory, Interscience, New York, 1981. Reprinted by Krieger, Malabar, Florida, 1993. MR0606200 (82j:41001)

16. Seidel, H.-P., Polar forms and triangular B-spline surfaces, in Blossoming: The New PolarForm Approach to Spline Curves and Surfaces SIGGRAPH '91, Course Notes \#26, ACM SIGGRAPH, 1991.

17. Shamos, M. I., and D. Hoey, Closest-point problems, in IEEE Symposium on Foundations of Computer Science, 1975, 151-162. MR0426498 (54:14441)

Department of Mathematics, Center for Constructive Approximation, Vanderbilt University, Nashville, Tennessee 37240

E-mail address: neamtu@math.vanderbilt.edu 